\title{
Numerical Solution of Fuzzy Differential Equations with Z-numbers using Fuzzy Sumudu Transforms
}

\author{
Sina Razvarz ${ }^{1}$, Raheleh Jafari ${ }^{*}, 2$, Wen $\mathrm{Yu}^{1}$ \\ ${ }^{1}$ Departamento de Control Automatico CINVESTAV-IPN (National Polytechnic Institute) Mexico City 07360, Mexico \\ ${ }^{2}$ Department of Information and Communication Technology Agder University College, 4876 Grimstad, Norway
}

\begin{tabular}{l} 
A R T I C L E I N F O \\
\hline Article history: \\
Received: 14 November, 2017 \\
Accepted: 17 January, 2017 \\
Online: 30 January, 2018 \\
\hline Keywords: \\
K fuzzy Sumudu transform \\
fuzzy differential equation \\
Z-number \\
\hline
\end{tabular}

\begin{abstract}
A B S T R A C T
The uncertain nonlinear systems can be modeled with fuzzy differential equations (FDEs) and the solutions of these equations are applied to analyze many engineering problems. However, it is very difficult to obtain solutions of FDEs.

In this paper, the solutions of FDEs are approximated by utilizing the fuzzy Sumudu transform (FST) method. Here, the uncertainties are in the sense of Z-numbers. Important theorems are laid down to illustrate the properties of FST. The theoretical analysis and simulation results show that this new technique is effective to estimate the solutions of FDES.
\end{abstract}

\section{Introduction}

This paper is an extension of work originally presented in [1]. In many physical and dynamical processes, mathematical modeling leads to the deterministic initial and boundary value problems. In practical the boundary values may be different from crisp and displays in the form of unknown parameters [2]. When the parameters or the states of the differential equations are uncertain, they can be modeled with FDE. In recent days, many methods have used FDE for modeling and control of uncertain nonlinear systems [3-5]. The basic idea of the fuzzy derivative was first introduced in [Chang]. Then it is extended in [6]. The firstorder fuzzy initial value problem, as well as fuzzy partial differential equation, have been studied in [7]. By generalizing the differentiability, [6] gave an analytical solution. The Lipschitz condition, as well as the theorem for existence and uniqueness of the solution related to FDEs, are discussed in [10-12]. In [13], the analytical solutions of second order FDE are obtained. The analytical solutions of third order linear FDE are found in [14]. By the interval-valued method, [15] examined the basic solutions of nonlinear FDEs with generalized differentiability.

A novel technique in order to solve FDEs is laid down based on the Sumudu transform. Sumudu transform along with broad applications has been utilized in the area of system engineering and applied physics [16-18]. In [19], some simple and deeper

\footnotetext{
*Corresponding Author: Raheleh Jafari, jafari3339@yahoo.com
}

fundamental theorems, as well as properties of the Sumudu Transform, were generalized. In [20], Sumudu transform is applied to the system of differential equations. In [21], Sumudu transform is used in order to find the solution of the fuzzy partial differential equation. In [22], Sumudu transform has been used to solve fractional differential equations.

In this paper, we use FST to approximate the Z-number solutions of the FDEs. The FST reduces the FDE to an algebraic equation. A very important property of the FST is that it can solve the equation without resorting to a new frequency domain. The procedure of switching FDEs to an algebraic equation is cited in [10] and is stated as an operational calculus. We extend our previous work [1] by generating more theorems for describing the properties of FST and displaying the uncertainties with Z-numbers. The Z-number is a new concept that is subjected to a higher potential to demonstrate the information of the human being as well as to utilize in information processing [23]. Z-numbers can be regarded as to answer questions and carry out the decisions [24]. There exist few structure based on the theoretical concept of Znumbers [25]. [26] gave an inception, which results in the extension of the Z-numbers. [27] generated a theorem to convert the Z-numbers to the usual fuzzy sets.

In this paper, initially, some preliminary definitions along with properties related to FST are demonstrated. After that, solving FDEs by using the methodology of FST has been discussed. At the end, two examples along with comparisons are utilized in order to demonstrate the effectiveness of our proposed method. 


\section{Preliminaries}

Prior to the introduction of the FST, some concepts related to the fuzzy variables and Z-numbers are laid down in this section $[28,29]$.

Definition 1: A fuzzy number $B$ is a function of $B \in E: R \rightarrow[0,1]$, in such a manner, 1$) B$ is normal, (there exists $a_{0} \in R$ in such a manner $B\left(a_{0}\right)=1$;2) $B$ is convex, $B(\gamma a+(1-\gamma) c) \geq \min \{B(a), B(c)\}, \forall a, c \in R, \forall \gamma \in[0,1]$

; 3) $B$ is upper semi-continuous on $R$, i.e., $B(a) \leq B\left(a_{0}\right)+\varepsilon$, $\forall a \in N\left(a_{0}\right), \forall a_{0} \in R, \forall \varepsilon>0, N\left(a_{0}\right)$ is a neighborhood; 4) The set $B^{+}=\{a \in R, B(a)>0\}$ is compact.

Definition 2: The $r$-level of the fuzzy number $B$ is defined as follows

$$
[B]^{r}=\{a \in R: B(a) \geq r\}
$$

where $0<r \leq 1, B \in E$.

Definition 3: Let $B_{1}, B_{2} \in E$ and $\xi \in R$, the operations addition, subtraction, multiplication and scalar multiplication are defined as

$$
\begin{gathered}
{\left[B_{1} \oplus B_{2}\right]^{r}=\left[B_{1}\right]^{r}+\left[B_{2}\right]^{r}=\left[\underline{B}_{1}^{r}+\underline{B}_{2}^{r}, \bar{B}_{1}^{r}+\bar{B}_{2}^{r}\right]} \\
{\left[B_{1} ? B_{2}\right]^{r}=\left[B_{1}\right]^{r}-\left[B_{2}\right]^{r}=\left[\underline{B}_{1}^{r}-\underline{B}_{2}^{r}, \bar{B}_{1}^{r}-\bar{B}_{2}^{r}\right]} \\
{\left[B_{1} ? B_{2}\right]^{r}=\left(\begin{array}{ll}
\min \left\{\underline{B}_{1}^{r} \underline{B}_{2}^{r}, \underline{B}_{1}^{r} \bar{B}_{2}^{r}, \bar{B}_{1}^{r} \underline{B}_{2}^{r}, \bar{B}_{1}^{r} \bar{B}_{2}^{r}\right\} \\
\max \left\{\underline{B}_{1}^{r} \underline{B}_{2}^{r}, \underline{B}_{1}^{r} \bar{B}_{2}^{r}, \bar{B}_{1}^{r} \underline{B}_{2}^{r}, \bar{B}_{1}^{r} \bar{B}_{2}^{r}\right\}
\end{array}\right)} \\
{\left[\xi B_{1}\right]^{r}=\xi\left[B_{1}\right]^{r}= \begin{cases}\left(\xi \underline{B}_{1}^{r}, \xi \bar{B}_{1}^{r}\right), & \xi \geq 0 \\
\left(\xi \bar{B}_{1}^{r}, \xi \underline{B}_{1}^{r}\right), & \xi \leq 0\end{cases} }
\end{gathered}
$$

Definition 4: The Hausdorff distance between two fuzzy numbers $B_{1}$ and $B_{2}$ is defined as [30,31]

$$
D\left(B_{1}, B_{2}\right)=\sup _{0 \leq r \leq 1}\left\{\max \left(\left|\underline{B}_{1}^{r}-\underline{B}_{2}^{r}\right|,\left|\bar{B}_{1}^{r}-\bar{B}_{2}^{r}\right|\right)\right\}
$$

$D\left(B_{1}, B_{2}\right)$ has the following properties

(i) $D\left(B_{1} \oplus u, B_{2} \oplus u\right)=D\left(B_{1}, B_{2}\right), \forall B_{1}, B_{2}, u \in E$

(ii) $D\left(\xi B_{1}, \xi B_{2}\right)=|\xi| D\left(B_{1}, B_{2}\right), \forall \xi \in R, B_{1}, B_{2} \in E$

(iii) $D\left(B_{1} \oplus B_{2}, u \oplus v\right) \leq D\left(B_{1}, u\right)+D\left(B_{2}, v\right)$,

$$
\forall B_{1}, B_{2}, u, v \in E
$$

(iv) $(D, E)$ is stated as complete metric space.
Definition 5: The function $\psi:\left[a_{1}, a_{2}\right] \rightarrow E$ is integrable on $\left[a_{1}, a_{2}\right]$, if it satisfies in the below mentioned relation

$$
\int_{a_{1}}^{\infty} \psi(x) d x=\left(\int_{a_{1}}^{\infty} \psi(x, r) d x, \int_{a_{1}}^{\infty} \bar{\psi}(x, r) d x\right)
$$

If $\psi(x)$ be a fuzzy value function, as well as $q(x)$ be a fuzzy Riemann integrable on $\left[a_{1}, \infty\right]$ so $\psi(x) \oplus q(x)$ can be a fuzzy Riemann integrable on $\left[a_{1}, \infty\right]$. Therefore,

$$
\int_{a_{1}}^{\infty}(\psi(x) \oplus q(x)) d x=\int_{a}^{\infty} \psi(x) d x \oplus \int_{a}^{\infty} q(x) d x
$$

According to the fuzzy concept or in the case of interval arithmetic, equation $B_{1}=B_{2} \oplus S$ is not equivalent with $s=B_{1} ? B_{2}=B_{1} \oplus(-1) B_{2}$ or to $B_{2}=B_{1} ? s=B_{1} \oplus(-1) s$ and this is the main reason in introducing the following Hukuhara difference (H-difference).

Definition 6: The definition of H-difference [32,9], is proposed by $B_{1} ?_{H} B_{2}=s \Leftrightarrow B_{1}=B_{2} \oplus s$. If $B_{1} ?_{H} B_{2}$ prevails, its $r$ level is $\left[B_{1} ?_{H} B_{2}\right]^{r}=\left[\underline{B}_{1}^{r}-\underline{B}_{2}^{r}, \bar{B}_{1}^{r}-\bar{B}_{2}^{r}\right]$.

Precisely, $B_{1} ?_{H} B_{1}=0$ but $B_{1} ? B_{1} \neq 0$.

Definition 7: Suppose $\psi:\left[a_{1}, a_{2}\right] \rightarrow E$ and $x_{0}=\left[a_{1}, a_{2}\right]$. $\psi$ is strongly generalized differentiable at $x_{0}$, if for all $k>0$ adequately minute, $\psi^{\prime}\left(x_{0}\right) \in E$ exists in such a manner that (i) $\exists \psi\left(x_{0}+k\right) ?_{H} \psi\left(x_{0}\right), \psi\left(x_{0}\right) ?_{H} \psi\left(x_{0}-k\right)$ and

$$
\begin{aligned}
& \lim _{k \rightarrow 0^{+}} \frac{\psi\left(x_{0}+k\right) ?_{H} \psi\left(x_{0}\right)}{k}=\lim _{k \rightarrow 0^{+}} \frac{\psi\left(x_{0}\right) ?_{H} \psi\left(x_{0}-k\right)}{k} \\
& =\psi^{\prime}\left(x_{0}\right)
\end{aligned}
$$

or (ii) $\exists \psi\left(x_{0}\right) ?_{H} \psi\left(x_{0}+k\right), \psi\left(x_{0}-k\right) ?_{H} \psi\left(x_{0}\right)$ and

$$
\begin{aligned}
& \lim _{k \rightarrow 0^{+}} \frac{\psi\left(x_{0}\right) ?_{H} \psi\left(x_{0}+k\right)}{(-k)}=\lim _{k \rightarrow 0^{+}} \frac{\psi\left(x_{0}-k\right) ?_{H} \psi\left(x_{0}\right)}{(-k)} \\
& =\psi^{\prime}\left(x_{0}\right), \\
& \text { or (iii) } \exists \psi\left(x_{0}+k\right) ?_{H} \psi\left(x_{0}\right), \psi\left(x_{0}-k\right) ?_{H} \psi\left(x_{0}\right) \text { and }
\end{aligned}
$$

$$
\begin{aligned}
& \lim _{k \rightarrow 0^{+}} \frac{\psi\left(x_{0}+k\right) ?_{H} \psi\left(x_{0}\right)}{k}=\lim _{k \rightarrow 0^{+}} \frac{\psi\left(x_{0}-k\right) ?_{H} \psi\left(x_{0}\right)}{(-k)} \\
& =\psi^{\prime}\left(x_{0}\right)
\end{aligned}
$$

or (iv) $\exists \psi\left(x_{0}\right) ?_{H} \psi\left(x_{0}+k\right), \psi\left(x_{0}\right) ?_{H} \psi\left(x_{0}-k\right)$ and

$$
\begin{aligned}
& \lim _{k \rightarrow 0^{+}} \frac{\psi\left(x_{0}\right) ?_{H} \psi\left(x_{0}+k\right)}{(-k)}=\lim _{k \rightarrow 0^{+}} \frac{\psi\left(x_{0}\right) ?_{H} \psi\left(x_{0}-k\right)}{k} \\
& =\psi^{\prime}\left(x_{0}\right)
\end{aligned}
$$

Remark 1:It is clear that case $(i)$ is H-derivative. Furthermore, a function is (i)-differentiable only when it is H-derivative. Remark 2: It can be concluded from [32] that, the definition of differentiability is noncontradictory [33]. 
Let us consider $\psi: R \rightarrow E$ where $\psi(t)$ has a parametric form as $[\psi(t, r)]=[\underline{\psi}(t, r), \bar{\psi}(t, r)]$, for all $0 \leq r \leq 1$, thus (i) If $\psi$ be (i)-differentiable, so $\underline{\psi}(t, r)$ and $\bar{\psi}(t, r)$ are differentiable functions, moreover $\psi^{\prime}(t)=\left(\underline{\psi}^{\prime}(t, r), \bar{\psi}^{\prime}(t, r)\right)$. (ii) If $\psi$ be (ii)-differentiable, so $\underline{\psi}(t, r)$ and $\bar{\psi}(t, r)$ are differentiable functions, moreover $\psi^{\prime}(t)=\left(\bar{\psi}^{\prime}(t, r), \underline{\psi^{\prime}}(t, r)\right)$. Suppose $f:\left(a_{1}, a_{2}\right) \rightarrow R$ is differentiable on $\left(a_{1}, a_{2}\right)$, furthermore $\psi^{\prime}$ has finite root in $\left(a_{1}, a_{2}\right)$, and $m \in E$, therefore, $\psi(x)=m f(x)$ is strongly generalized differentiable on $\left(a_{1}, a_{2}\right)$ along with $\psi^{\prime}(x)=m f^{\prime}(x), \forall x \in\left(a_{1}, a_{2}\right)$.

Theorem 1: [9] Assume $\psi: R \times E \rightarrow E$ is taken to be a continuous fuzzy function. If $x_{0} \in R$, the fuzzy initial value constraint

$$
\left\{\begin{array}{c}
\phi^{\prime}(t)=\psi(x, \phi) \\
\phi\left(x_{0}\right)=\phi_{0}
\end{array}\right.
$$

is incorporated with two solutions: (i)-differentiable, also (ii)differentiable. Hence the successive iterations

$$
\phi_{n+1}(x)=\phi_{0}+\int_{x_{0}}^{x} \psi\left(t, \phi_{n}(t)\right) d t, \quad \forall x \in\left[x_{0}, x_{1}\right]
$$

and

$$
\varphi_{n+1}(x)=\varphi_{0} ?_{H}(-1) \int_{x_{0}}^{x} \psi\left(t, \varphi_{n}(t)\right) d t, \quad \forall x \in\left[x_{0}, x_{1}\right]
$$

approaches towards the two solutions sequentially.

Definition 8: A Z-number has two components $Z=[B(a), \tilde{p}]$. The primary component $B(a)$ is restriction on a real-valued uncertain variable $a$. The secondary component $\tilde{p}$ is a measure of the reliability of $B . \tilde{p}$ can be reliability, the strength of belief, probability or possibility. When $B(a)$ is a fuzzy number and $\widetilde{p}$ is the probability distribution of $a$, the Z-number is stated as $Z^{+}$number. When $B(a)$ as well as $\tilde{p}$ are fuzzy numbers, the Znumber is stated as $Z^{-}$-number.

The $Z^{+}$-number carries more information when compared with $Z^{-}$-number. In this paper, we utilize the definition of $Z^{+}$number, i.e., $Z=[B, \widetilde{p}], \quad B$ is a fuzzy number and $\widetilde{p}$ is a probability distribution.

To express the fuzzy number the most common membership functions are utilized in this paper. The popular membership functions are the triangular function

$\mu_{B}=F\left(\lambda_{1}, \lambda_{2}, \lambda_{3}\right)=\left\{\begin{array}{ll}\frac{a-\lambda_{1}}{\lambda_{2}-\lambda_{1}} & \lambda_{1} \leq a \leq \lambda_{2} \\ \frac{\lambda_{3}-\zeta}{\lambda_{3}-\lambda_{2}} & \lambda_{2} \leq a \leq \lambda_{3}\end{array}\right.$ otherwise $\mu_{B}=0$ (12) and trapezoidal function

$$
\mu_{B}=F\left(\lambda_{1}, \lambda_{2}, \lambda_{3}, \lambda_{4}\right)=\left\{\begin{array}{cl}
\frac{a-\lambda_{1}}{\lambda_{2}-\lambda_{1}} & \lambda_{1} \leq a \leq \lambda_{2} \\
\lambda_{4}-a & \lambda_{3} \leq a \leq \lambda_{4} \text { otherwise } \mu_{B}=0 \\
1 & \lambda_{2} \leq a \leq \lambda_{3}
\end{array}\right.
$$

The probability measure is defined as

$$
\widetilde{P}=\int_{R} \mu_{B}(a) \tilde{p}(a) d a
$$

where $\widetilde{p}$ is the probability density of $a$, also $R$ is the restriction on $\tilde{p}$. For discrete Z-numbers

$$
\widetilde{P}(B)=\sum_{i=1}^{n} \mu_{B}\left(a_{i}\right) \widetilde{p}\left(a_{i}\right)
$$

Definition 9: The $r$-level of the Z-number $Z=(B, \widetilde{P})$ is illustrated as

$$
[Z]^{r}=\left([B]^{r},[\tilde{p}]^{r}\right)
$$

where $0<r \leq 1$. [ $\tilde{p}]^{r}$ is computed by the Nguyen's theorem

$$
[\widetilde{p}]^{r}=\widetilde{p}\left([B]^{r}\right)=\widetilde{p}\left(\left[\underline{B}^{r}, \bar{B}^{r}\right]\right)=\left[\underline{\widetilde{P}}^{r}, \overline{\widetilde{P}}^{r}\right]
$$

where $\tilde{p}\left([B]^{r}\right)=\left\{\tilde{p}(a) \mid a \in[B]^{r}\right\}$. So $[Z]^{r}$ can be demonstrated as the form $r$-level of a fuzzy number

$$
[Z]^{r}=\left(\underline{Z}^{r}, \bar{Z}^{r}\right)=\left(\left(\underline{B}^{r}, \underline{\widetilde{P}}^{r}\right),\left(\bar{B}^{r}, \overline{\widetilde{P}}^{r}\right)\right)
$$

where $\underline{\widetilde{P}}^{r}=\underline{B}^{r} \tilde{p}\left({\underline{a_{i}}}^{r}\right), \overline{\widetilde{P}}^{r}=\bar{B}^{r} \widetilde{p}\left({\overline{a_{i}}}^{r}\right),\left[a_{i}\right]^{r}=\left(\underline{a_{i}},{\overline{a_{i}}}^{r}\right)$.

Similar with the fuzzy numbers, the Z-numbers are also incorporated with three primary operations, addition, subtraction, and multiplication. The operations in this paper are different definitions with [34]. The $r$-level of Z-numbers is applied to simplify the operations.

Suppose $Z_{1}=\left(B_{1}, \widetilde{p}_{1}\right)$ and $Z_{2}=\left(B_{2}, \widetilde{p}_{2}\right)$ be two discrete Znumbers expressing the uncertain variables $a_{1}$ and $a_{2}$, $\sum_{l=1}^{n} \widetilde{p}_{1}\left(a_{1 l}\right)=1, \quad \sum_{l=1}^{n} \widetilde{p}_{2}\left(a_{2 l}\right)=1$. The operations are displayed as

$$
Z_{12}=Z_{1} * Z_{2}=\left(B_{1} * B_{2}, \tilde{p}_{1} * \tilde{p}_{2}\right)
$$

where $* \in\{\oplus, ?, ?\}$.

For all $\widetilde{p}_{1} * \widetilde{p}_{2}$ operations, we use convolutions for the discrete probability distributions

$$
\widetilde{p}_{1} * \widetilde{p}_{2}=\sum_{i} \widetilde{p}_{1}\left(a_{1, i}\right) \widetilde{p}_{2}\left(a_{2,(n-i)}\right)=\widetilde{p}_{12}(a)
$$

The above definitions satisfy the Hukuhara difference [35],

$$
\begin{aligned}
& Z_{1}{ }_{H} Z_{2}=Z_{12} \\
& Z_{1}=Z_{2} \oplus Z_{12}
\end{aligned}
$$


If $Z_{1} ?_{H} Z_{2}$ prevails, the $r$-level is

$$
\left[Z_{1} ?_{H} Z_{2}\right]^{r}=\left[\underline{Z}_{1}^{r}-\underline{Z}_{2}^{r}, \bar{Z}_{1}^{r}-\bar{Z}_{2}^{r}\right]
$$

Obviously, $Z_{1} ?_{H} Z_{1}=0, Z_{1} ? Z_{1} \neq 0$.

Also the above definitions satisfy the generalized Hukuhara difference [7]

$$
Z_{1} ?_{g H} Z_{2}=Z_{12} \Leftrightarrow\left\{\begin{array}{l}
\text { 1) } Z_{1}=Z_{2} \oplus Z_{12} \\
\text { 2) } Z_{2}=Z_{1} \oplus(-1) Z_{12}
\end{array}\right.
$$

It is easy to display that 1) and 2) in combination are genuine if and only if $Z_{12}$ is a crisp number. With respect to $r$-level we have

$\left[Z_{1} ?_{g H} Z_{2}\right]^{r}=\left[\min \left\{\underline{Z}_{1}^{r}-\underline{Z}_{2}^{r}, \bar{Z}_{1}^{r}-\bar{Z}_{2}^{r}\right\}, \max \left\{\underline{Z}_{1}^{r}-\underline{Z}_{2}^{r}, \bar{Z}_{1}^{r}-\bar{Z}_{2}^{r}\right\}\right]$

and If and subsist, $Z_{1} ?_{H} Z_{2}=Z_{1} ?_{g H} Z_{2}$. The circumstances for the inerrancy of $Z_{12}=Z_{1} ?_{g H} Z_{2} \in E$ are

1) $\left\{\begin{array}{c}\underline{Z}_{12}^{r}=\underline{Z}_{1}^{r}-\underline{Z}_{2}^{r} \text { and } \bar{Z}_{12}^{r}=\bar{Z}_{1}^{r}-\bar{Z}_{2}^{r} \\ \text { with } \underline{Z}_{12}^{r} \text { increa } \sin g, \bar{Z}_{12}^{r} \text { decrea } \sin g, \underline{Z}_{12}^{r} \leq \bar{Z}_{12}^{r}\end{array}\right.$

2) $\left\{\begin{array}{c}\underline{Z}_{12}^{r}=\bar{Z}_{1}^{r}-\bar{Z}_{2}^{r} \text { and } \bar{Z}_{12}^{r}=\underline{Z}_{1}^{r}-\underline{Z}_{2}^{r} \\ \text { with } \underline{Z}_{12}^{r} \text { increa } \sin g, \bar{Z}_{12}^{r} \text { decrea } \sin g, \underline{Z}_{12}^{r} \leq \bar{Z}_{12}^{r}\end{array}\right.$

where $\forall r \in[0,1]$

If $B$ is a triangular function, the absolute value of the $Z$-number $Z=(B, \tilde{p})$ is defined as

$$
|Z(a)|=\left(\left|\lambda_{11}\right|+\left|\lambda_{12}\right|+\left|\lambda_{13}\right|, \widetilde{p}\left(\left|\lambda_{21}\right|+\left|\lambda_{22}\right|+\left|\lambda_{23}\right|\right)\right)
$$

If $B_{1}$, as well as $B_{2}$ are triangular functions, the supremum metric for $Z$-numbers $Z_{1}=\left(B_{1}, \widetilde{p}_{1}\right)$ and $Z_{2}=\left(B_{2}, \tilde{p}_{2}\right)$ is illustrated as

$$
D\left(Z_{1}, Z_{2}\right)=d\left(B_{1}, B_{2}\right)+d\left(\widetilde{p}_{1}, \widetilde{p}_{2}\right)
$$

in this case $d(\cdot, \cdot)$ is defined as the supremum metrics with fuzzy sets [3]. $D\left(Z_{1}, Z_{2}\right)$ is incorporated with the following possessions

$$
\begin{aligned}
& D\left(Z_{1}+Z, Z_{2}+Z\right)=D\left(Z_{1}, Z_{2}\right) \\
& D\left(Z_{2}, Z_{1}\right)=D\left(Z_{1}, Z_{2}\right) \\
& D\left(Z_{1}, \zeta Z_{2}\right)=|\zeta| D\left(Z_{1}, Z_{2}\right) \\
& D\left(Z_{1}, Z_{2}\right) \leq D\left(Z_{1}, Z\right)+D\left(Z, Z_{2}\right)
\end{aligned}
$$

where $\zeta \in R, Z=(B, \widetilde{p})$ is Z-number and $B$ is triangle function, for proof refer to [36].

Definition 10: Suppose $\hat{Z}$ demonstrates the space of $Z$ numbers, then the $r-$ level of $Z$-number valued function $\Psi:\left[a_{1}, a_{2}\right] \rightarrow \hat{Z}$ is defined as

$$
\Psi(\phi, r)=[\underline{\Psi}(\phi, r), \bar{\Psi}(\phi, r)]
$$

where $\phi \in \hat{Z}$, and $r \in[0,1]$.

Based on the definition of Generalized Hukuhara difference, the gH-derivative of $\Psi$ at $\phi_{0}$ is defined as

$$
\Psi^{\prime}\left(\varphi_{0}\right)=\lim _{h \rightarrow 0} \frac{1}{h}\left[\Psi\left(\varphi_{0}+h\right) ?_{g H} \Psi\left(\varphi_{0}\right)\right]
$$

In (27), $\Psi\left(\phi_{0}+h\right)$ and $\Psi\left(\phi_{0}\right)$ represents similar style with $Z_{1}$ and $Z_{2}$ respectively defined in (laiop).

By implementing the $r$ - level (16) to initial value problem, $\phi^{\prime}(t)=\psi(t, \phi(t))$, we generate two Z-number valued functions: $\underline{\psi}[t, \underline{\phi}(a, r), \bar{\phi}(a, r)]$ and $\bar{\psi}[t, \underline{\phi}(a, r), \bar{\phi}(a, r)]$ relation

The initial value problem can be equivalent to the following

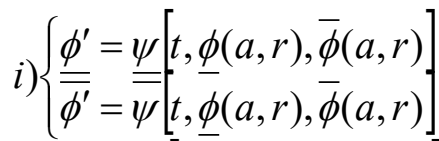

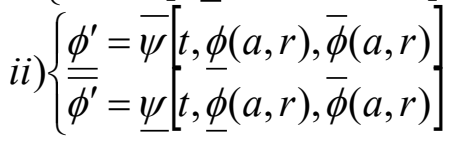

\section{Fuzzy Sumudu transform}

Fuzzy initial and boundary value problems can be resolved by utilizing fuzzy Laplace transform [10]. In this paper, the FST methodology for Z-number is illustrated, furthermore the properties of this methodology is stated. By applying the FST methodology, the FDE based on Z-number is reduced to an algebraic equation. The main advantage of the FST is that it can resolve the equation without resorting to a new frequency domain. The methodology of converting FDEs to an algebraic equation is expressed in [10].

Definition 11: Suppose $\psi(t)$ be a continuous Z-number valued function, also, $\psi(B t) ? e^{-t}$ be an improper Z-number Riemann integrable on $[0, \infty)$. Accordingly, $\int_{0}^{\infty} \psi(B t) ? e^{-t} d t$ is expressed as FST and it is defined by $\Omega(B)=\mathbf{S}[\psi(t)]=\int_{0}^{\infty} \psi(B t) ? e^{-t} d t$, where $0 \leq B<K, K \geq 0$ , also $e^{-t}$ is a real-valued function. Based on the Theorem 3 we have the following relation

$$
\int_{0}^{\infty} \psi(B t) ? e^{-t} d t=\left(\int_{0}^{\infty} \underline{\psi}(B t, r) e^{-t} d t, \int_{0}^{\infty} \bar{\psi}(B t, r) e^{-t} d t\right)
$$

Let

$$
\begin{aligned}
& \mathbf{S}[\underline{\psi}(t, r)]=\int_{0}^{\infty} \underline{\bar{\psi}}(B t, r) e^{-t} d t \\
& \mathbf{S}[\overline{\bar{\psi}}(t, r)]=\int_{0}^{\infty} \overline{\bar{\psi}}(B t, r) e^{-t} d t
\end{aligned}
$$

hence we obtain the following relation

$$
\mathbf{S}[\psi(t)]=(\mathbf{S}[\underline{\psi}(t, r), \mathbf{S} \bar{\psi}(t, r)])
$$


Theorem 2: Suppose $\psi^{\prime}(t)$ be a Z-number valued integrable function, as well as $\psi(t)$ be the primitive of $\psi^{\prime}(t)$ on $[0, \infty)$. Therefore,

$$
\mathbf{S}\left[\psi^{\prime}(t)\right]=\frac{1}{B} ? \mathbf{S}[\psi(t)] ?\left(\frac{1}{B} ?[\psi(0)]\right)
$$

where is considered to be (i)-differentiable, or

$$
\mathbf{S}\left[\psi^{\prime}(t)\right]=\frac{-1}{B} ?[\psi(0)] ?\left(\frac{-1}{B} ? \mathbf{S}[\psi(t)]\right)
$$

where $\psi$ is considered to be (ii)-differentiable.

Proof. For arbitrary fixed $r \in[0,1]$ we have

$$
\begin{aligned}
& \frac{1}{B} ? \mathbf{S}[\psi(t)] ?\left(\frac{1}{B} ? \psi(0)\right) \\
& =\left(\frac{1}{B} \mathbf{S}[\underline{\psi}(t, r)]-\frac{1}{B} \mathbf{S}[\underline{\psi}(0, r)], \frac{1}{B} \mathbf{S}[\bar{\psi}(t, r)]-\frac{1}{B} \mathbf{S}[\bar{\psi}(0, r)]\right)
\end{aligned}
$$

We have the following relations

$$
\begin{aligned}
& \mathbf{S}\left[\bar{\psi}^{\prime}(t, r)\right]=\frac{1}{B} \mathbf{S}[\bar{\psi}(t, r)]-\frac{1}{B}[\bar{\psi}(0, r)] \\
& \mathbf{S}\left[\underline{\psi^{\prime}}(t, r)\right]=\frac{1}{B} \mathbf{S}\left[\underline{\psi(t, r)]-\frac{1}{B}[\underline{\psi(0, r)]}}\right.
\end{aligned}
$$

Hence, we obtain

$$
\frac{1}{B} ? \mathbf{S}[\psi(t)] ?\left(\frac{1}{B} ? \psi(0)\right)=\left(\mathbf{S}\left[\underline{\psi}^{\prime}(t, r)\right], \mathbf{S}\left[\bar{\psi}^{\prime}(t, r)\right]\right)
$$

If is considered to be (i)-differentiable, so

$$
\frac{1}{B} ? \mathbf{S}[\psi(t)] ?\left(\frac{1}{B} ? \psi(0)\right)=\mathbf{S}\left[\psi^{\prime}(t)\right]
$$

Let is (ii)-differentiable. For arbitrary fixed $\alpha \in[0,1]$ we

$$
\text { obtain } \begin{aligned}
& \frac{-1}{B} ?[\psi(0)] ?\left(\frac{-1}{B} ? \mathbf{S}[\psi(t)]\right) \\
& =\left(\frac{-1}{B} \bar{\psi}(0, r)+\frac{1}{B} \mathbf{S}[\bar{\psi}(t, r)], \frac{-1}{B} \underline{\psi}(0, r)+\frac{1}{B} \mathbf{S}[\underline{\psi}(t, r)]\right)
\end{aligned}
$$

The above equation can be written as the following relation

$$
\begin{aligned}
& \frac{-1}{B} ?[\psi(0)] ?\left(\frac{-1}{B} ? \mathbf{S}[\psi(t)]\right) \\
& =\left(\frac{1}{B} \mathbf{S}[\bar{\psi}(t, r)]-\frac{1}{B} \bar{\psi}(0, r), \frac{1}{B} \mathbf{S}[\underline{\psi}(t, r)]-\frac{1}{B} \underline{\psi}(0, r)\right)
\end{aligned}
$$

We obtain

$$
\begin{aligned}
& \mathbf{S}\left[\bar{\psi}^{\prime}(t, r)\right]=\frac{1}{B} \mathbf{S}[\bar{\psi}(t, r)]-\frac{1}{B} \bar{\psi}(0, r) \\
& \mathbf{S}\left[\underline{\psi^{\prime}}(t, r)\right]=\frac{1}{B} \mathbf{S}[\underline{\psi}(t, r)]-\frac{1}{B} \underline{\psi}(0, r)
\end{aligned}
$$

So, we have

$$
\left(\frac{-1}{B} \psi(0)\right) ?\left(\frac{-1}{B} ? \mathbf{S}[\psi(t)]\right)=\left(\mathbf{S}\left[\bar{\psi}^{\prime}(t, r)\right], \mathbf{S}\left[\underline{\psi}^{\prime}(t, r)\right]\right)
$$

Hence

$$
\left(\frac{-1}{B} \psi(0)\right) ?\left(\frac{-1}{B} ? \mathbf{S}[\psi(t)]\right)=\mathbf{S}\left(\left[\bar{\psi}^{\prime}(t, r)\right],\left[\underline{\psi}^{\prime}(t, r)\right]\right)
$$

Since $\psi$ is (ii)-differentiable, therefore,

$$
\left(\frac{-1}{B} \psi(0)\right) ?\left(\frac{-1}{B} ? \mathbf{S}[\psi(t)]\right)=\mathbf{S}\left[\psi^{\prime}(t)\right]
$$

Theorem 3: Taking into consideration that Sumudu transform is a linear transformation, so if $\psi(t)$ and $\vartheta(t)$ be continuous Znumber valued functions, moreover $k_{1}$ as well as $k_{2}$ be constant, therefore the following relation can be obtained

$$
\mathbf{S}\left[\left(k_{1} ? \psi(t)\right) \oplus\left(k_{2} ? \vartheta(t)\right)\right]=\left(k_{1} ? \mathbf{S}[\psi(t)]\right) \oplus\left(k_{2} ? \mathbf{S}[\vartheta(t)]\right)
$$

Proof. We have

$$
\begin{aligned}
& \mathbf{S}\left[\left(k_{1} ? \psi(t)\right) \oplus\left(k_{2} ? \vartheta(t)\right)\right] \\
& =\int_{0}^{\infty}\left(k_{1} ? \phi(B t) \oplus k_{2} ? \vartheta(B t)\right) ? e^{-t} d t \\
& =\int_{0}^{\infty} k_{1} ? \psi(B t) ? e^{-t} d t \oplus \int_{0}^{\infty} k_{2} ? \vartheta(B t) ? e^{-t} d t \\
& =k_{1} ?\left(\int_{0}^{\infty} \psi(B t) ? e^{-t} d t\right) \oplus k_{2} ?\left(\int_{0}^{\infty} \vartheta(B t) ? e^{-t} d t\right) \\
& =k_{1} ? \mathbf{S}[\psi(t)] \oplus k_{2} ? \mathbf{S}[\vartheta(t)]
\end{aligned}
$$

Therefore, we conclude

$$
\mathbf{S}\left[\left(k_{1} ? \psi(t)\right) \oplus\left(k_{2} ? \vartheta(t)\right)\right]=\left(k_{1} ? \mathbf{S}[\psi(t)]\right) \oplus\left(k_{2} ? \mathbf{S}[\vartheta(t)]\right)
$$

Lemma 1: Assume that $\psi(t)$ is a continuous Z-number value function on $[0, \infty)$, also $\gamma \geq 0$, thus

$$
\mathbf{S}[\gamma ? \psi(t)]=\gamma ? \mathbf{S}[\psi(t)]
$$

Proof. Fuzzy Sumudu transform $\gamma ? \psi(t)$ is defined as

$$
\mathbf{S}[\gamma ? \psi(t)]=\int_{0}^{\infty} \gamma ? \psi(B t) ? e^{-t} d t
$$

furthermore, we have

$$
\int_{0}^{\infty} \gamma ? \psi(B t) ? e^{-t} d t=\gamma ? \int_{0}^{\infty} \psi(B t) ? e^{-t} d t
$$

therefore,

$$
\mathbf{S}[\gamma ? \psi(t)]=\gamma ? \mathbf{S}[\psi(t)]
$$

Lemma 2: Assume that $\psi(t)$ is a continuous Z-number valued function, and $\vartheta(t) \geq 0$. Furthermore, if we suppose that $(\psi(t) ? \vartheta(t)) ? e^{-t}$ is improper Z-number Reiman integrable on $[0, \infty)$, then

$$
\begin{aligned}
& \int_{0}^{\infty}(\psi(B t) ? \vartheta(B t)) ? e^{-t} d t \\
& =\left(\int_{0}^{\infty} \vartheta(B t) \underline{\psi}(B t, r) e^{-t} d t, \int_{0}^{\infty} \vartheta(B t) \bar{\psi}(B t, r) e^{-t} d t\right)
\end{aligned}
$$

Theorem 4: Suppose $\psi(t)$ is a continuous Z-number valued function, also $\mathbf{S}[\psi(t)]=D(B)$, therefore,

$$
\mathbf{S}\left[e^{a_{1} t} ? \psi(t)\right]=\frac{1}{1-a_{1} B} D\left(\frac{B}{1-a_{1} B}\right)
$$

where $e^{a_{1} t}$ is considered to be a real value function, also $1-a_{1} B>0$. 
Proof. We have the following relation

$$
\begin{aligned}
& \mathbf{S}\left[e^{a_{1} t} ? \psi(t)\right]=\int_{0}^{\infty} e^{a_{1} B t} e^{-t} \psi(B t) d t \\
& =\left(\int_{0}^{\infty} e^{-\left(1-a_{1} B\right) t} \underline{\psi}(B t, r) d t, \int_{0}^{\infty} e^{-\left(1-a_{1} B\right) t} \bar{\psi}(B t, r) d t\right)
\end{aligned}
$$

Let us consider $z=1-a_{1} B t$, then

$$
\begin{aligned}
& \mathbf{S}\left[e^{a_{1} t} ? \psi(t)\right] \\
& =\frac{1}{1-a_{1} B}\left(\int_{0}^{\infty} \underline{\psi}\left(\frac{B z}{1-a_{1} B}, r\right) e^{-z} d z, \int_{0}^{\infty} \bar{\psi}\left(\frac{B z}{1-a_{1} B}, r\right) e^{-z} d z\right) \\
& =\left\{\frac{1}{1-a_{1} B} \underline{D}\left(\frac{B}{1-a_{1} B}\right), \frac{1}{1-a_{1} B} \bar{D}\left(\frac{B}{1-a_{1} B}\right)\right\}=\frac{1}{1-a_{1} B} D\left(\frac{B}{1-a_{1} B}\right)
\end{aligned}
$$

\section{Solving fuzzy initial value problem with fuzzy Sumudu transform method}

Consider the following fuzzy initial value problem based on Znumbers

$$
\left\{\begin{array}{l}
\phi^{\prime}(t)=\psi(t, \phi(t)), \\
\phi(0)=(\underline{\phi}(0, r), \bar{\phi}(0, r)), \quad 0<r \leq 1
\end{array}\right.
$$

where $\psi(t, \phi(t))$ is a Z-number function. By utilizing FST method for Z-numbers, we obtain

$$
\mathbf{S}\left[\phi^{\prime}(t)\right]=\mathbf{S}[\psi(t, \phi(t))]
$$

The solving process of Eq. (56) is based on the following cases.

Case 1: Assume that $\phi^{\prime}(t)$ is (i)-differentiable. Base on the Theorem 3 we extract

$$
\begin{gathered}
\phi^{\prime}(t)=\left(\underline{\phi^{\prime}}(t, r), \bar{\phi}^{\prime}(t, r)\right) \\
\mathbf{S}\left[\varphi^{\prime}(t)\right]=\left(\frac{1}{B} ? \mathbf{S}[\varphi(t)]\right) ? \frac{1}{B} \varphi(0)
\end{gathered}
$$

Eq. (58) can be displayed as the following relation

$$
\left\{\begin{array}{l}
\mathbf{S}[\underline{\psi}(t, \phi(t), r)]=\frac{1}{B} \mathbf{S}[\phi(t, r)]-\frac{1}{B} \phi(0, \alpha) \\
\mathbf{S}[\overline{\bar{\psi}}(t, \phi(t), r)]=\frac{1}{B} \mathbf{S}[\phi(t, r)]-\frac{1}{B} \bar{\phi}(0, r)
\end{array}\right.
$$

where

$$
\left\{\begin{array}{l}
\underline{\psi}(t, \phi(t), r)=\min \{\psi(t, B) \mid B \in(\underline{\phi}(t, r), \bar{\phi}(t, r))\} \\
\bar{\psi}(t, \phi(t), r)=\max \{\psi(t, B) \mid B \in(\underline{\phi}(t, r), \bar{\phi}(t, r))\}
\end{array}\right.
$$

Accordingly, Eq. (60) can be resolved on the basis of the following assumptions

$$
\begin{aligned}
& \mathbf{S}[\underline{\phi}(t, r)]=U_{1}(B, r) \\
& \mathbf{S}[\bar{\phi}(t, r)]=U_{2}(B, r)
\end{aligned}
$$

where $U_{1}(B, r)$, as well as $U_{2}(B, r)$ are the Z-number solutions of the Eq. (60). By applying inverse Sumudu transform, $\phi(t, r)$ as well as $\bar{\phi}(t, r)$ are computed as

$$
\begin{aligned}
\underline{\phi}(t, r) & =\mathbf{S}^{-1}\left[U_{1}(B, r)\right] \\
\bar{\phi}(t, r) & =\mathbf{S}^{-1}\left[U_{2}(B, r)\right]
\end{aligned}
$$

Case 2: Assume that $\phi^{\prime}(t)$ is (ii)-differentiable. Based on the Theorem 3 we extract

$$
\begin{gathered}
\phi^{\prime}(t)=\left(\bar{\phi}^{\prime}(t, r), \underline{\phi^{\prime}}(t, r)\right) \\
\mathbf{S}\left[\varphi^{\prime}(t)\right]=\left(\frac{-1}{B} ? \varphi(0)\right) ?\left(\frac{-1}{B} ? \mathbf{S}[\varphi(t)]\right)
\end{gathered}
$$

Eq. (66) can be displayed as the following relation

$$
\left\{\begin{array}{l}
\mathbf{S}[\underline{\underline{\psi}}(t, \phi(t), r)]=\frac{1}{B} \mathbf{S}[\underline{\phi}(t, r)]-\frac{1}{B} \phi(0, r) \\
\mathbf{S}[\psi(t, \phi(t), r)]=\frac{1}{B} \mathbf{S}[\overline{\bar{\phi}}(t, r)]-\frac{1}{B} \overline{\bar{\phi}}(0, r)
\end{array}\right.
$$

where

$$
\left\{\begin{array}{l}
\underline{\psi}(t, \phi(t), r)=\min \{\psi(t, B) \mid B \in(\underline{\phi}(t, r), \bar{\phi}(t, r))\} \\
\bar{\psi}(t, \phi(t), r)=\max \{\psi(t, B) \mid B \in(\underline{\phi}(t, r), \bar{\phi}(t, r))\}
\end{array}\right.
$$

Accordingly, Eq. (68) can be resolved on the basis of the following assumptions

$$
\begin{aligned}
& \mathbf{S}\left(\phi(t, r)=V_{1}(B, r)\right. \\
& \mathbf{S}\left(\phi(t, r)=V_{2}(B, r)\right.
\end{aligned}
$$

where $V_{1}(B, r)$, and $V_{2}(B, r)$ are the Z-number solutions of the Eq. (68). By applying inverse Sumudu transform, $\phi(t, r)$ as well as $\bar{\phi}(t, r)$ are computed as

$$
\begin{aligned}
& \phi(t, r)=\mathbf{S}^{-1}\left[V_{1}(B, r)\right] \\
& \overline{\bar{\phi}}(t, r)=\mathbf{S}^{-1}\left[V_{2}(B, r)\right]
\end{aligned}
$$

\section{Examples}

The following examples have been used to narrate the methodology proposed in this paper.

\subsection{Example 1}

A tank with a heating system is displayed in Figure 1, where $\widetilde{R}=0.5$, the thermal capacitance is $\widetilde{C}=2$ also the temperature is $\psi$. The model is formulated as follows $[10,37]$,

$$
\left\{\begin{array}{l}
\phi^{\prime}(t)=-\frac{1}{\widetilde{R} \widetilde{C}} \phi(t), \quad 0 \leq t \leq T \\
\phi(0)=[(\underline{\phi}(0, r), \bar{\phi}(0, r)), p(0.8,0.9,1)]
\end{array}\right.
$$

By utilizing the FST method based on Z-number we obtain

$$
\begin{aligned}
& \mathbf{S}\left[\phi^{\prime}(t)\right]=\mathbf{S}[-\phi(t)] \\
& S\left[\phi^{\prime}(B t)\right] \odot e^{-t} d t
\end{aligned}
$$

where $0 \leq B<K$. By considering case 1 for Z-numbers the following relation is obtained 


$$
\mathbf{S}\left[\varphi^{\prime}(t)\right]=\frac{1}{B} ?(\mathbf{S}[\varphi(t)] ? \varphi(0))=\frac{1}{B} \mathbf{S}[\varphi(t)] ? \frac{1}{B} \varphi(0)(74)
$$

Therefore

$$
-\mathbf{S}[\varphi(t)]=\frac{1}{B} \mathbf{S}[\varphi(t)] ? \frac{1}{B} \varphi(0)
$$

Based on the Eq. (59), we have

$$
\left\{\begin{array}{l}
-\mathbf{S}[\bar{\phi}(t, r)]=\frac{1}{B} \mathbf{S}[\underline{\phi}(t, r)]-\frac{1}{B} \underline{\bar{\phi}}(0, r) \\
-\mathbf{S}[\underline{\bar{\phi}}(t, r)]=\frac{1}{B} \mathbf{S}[\bar{\phi}(t, r)]-\frac{1}{B} \bar{\phi}(0, r)
\end{array}\right.
$$

Therefore, the Z-number solution of Eq. (76) is extracted as $[(\mathbf{S}[\bar{\phi}(t, r)], \mathbf{S}[\phi(t, r)]), p(0.8,0.94,1)]$ where

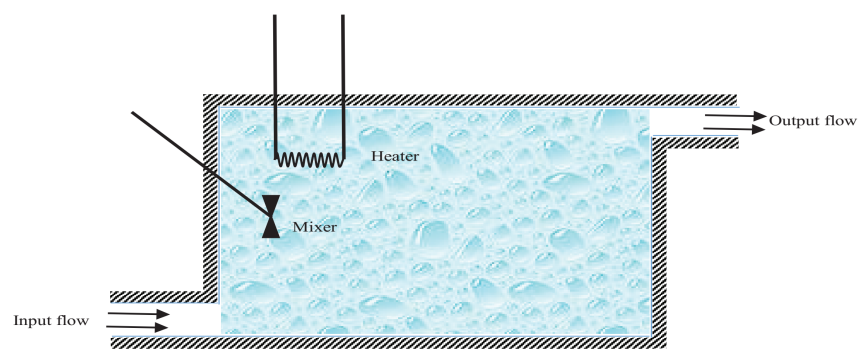

Fig. 1. A tank with a heating system

$$
\left\{\begin{array}{l}
\mathbf{S}[\bar{\phi}(t, r)]=\left(\frac{-1}{B^{2}-1}\right) \bar{\phi}(0, r)+\left(\frac{B}{B^{2}-1}\right) \phi(0, r) \\
\mathbf{S}[\underline{\phi}(t, r)]=\left(\frac{-1}{B^{2}-1}\right) \underline{\phi}(t, r)+\left(\frac{B}{B^{2}-1}\right) \bar{\phi}(0, r)
\end{array}\right.
$$

By utilizing the inverse Sumudu transform for Z-numbers, we have

$$
\left\{\begin{array}{l}
\mathbf{S}[\bar{\phi}(t, r)]=\bar{\phi}(0, r) \mathbf{S}^{-1}\left(\frac{-1}{B^{2}-1}\right)+\phi(0, r) \mathbf{S}^{-1}\left(\frac{B}{B^{2}-1}\right) \\
\mathbf{S}[\underline{\phi}(t, r)]=\underline{\phi}(0, r) \mathbf{S}^{-1}\left(\frac{-1}{B^{2}-1}\right)+\bar{\phi}(0, r) \mathbf{S}^{-1}\left(\frac{B}{B^{2}-1}\right)
\end{array}\right.
$$

where

$$
\left\{\begin{array}{l}
\bar{\phi}(t, r)=e^{t}\left(\frac{\bar{\phi}(0, r)-\underline{\underline{\phi}}(0, r)}{2}\right)+e^{-t}\left(\frac{\bar{\phi}(0, r)+\underline{\underline{\phi}}(0, r)}{2}\right) \\
\underline{\phi}(t, r)=e^{t}\left(\frac{\underline{\phi}(0, r)-\bar{\phi}(0, r)}{2}\right)+e^{-t}\left(\frac{\underline{\phi}(0, r)+\bar{\phi}(0, r)}{2}\right)
\end{array}\right.
$$

By considering case 2 for Z-numbers the following relation is obtained

$$
\mathbf{S}\left[\varphi^{\prime}(t)\right]=\left(\frac{-1}{B} \mathbf{S}[\varphi(t)]\right) ?\left(\frac{-1}{B} \varphi(0)\right)
$$

Hence

$$
-\mathbf{S}[\varphi(t)]=\left(\frac{-1}{B} \mathbf{S}[\varphi(t)]\right) ?\left(\frac{-1}{B} \varphi(0)\right)
$$

Based on the above relations, Eq. (71) is illustrated as

$$
\left\{\begin{array}{l}
-\mathbf{S}[\underline{\phi}(t, r)]=\frac{1}{B} \mathbf{S}[\underline{\phi}(t, r)]-\frac{1}{B} \phi(0, r) \\
-\mathbf{S}[\phi(t, r)]=\frac{1}{B} \mathbf{S}[\overline{\bar{\phi}}(t, r)]-\frac{1}{B} \overline{\bar{\phi}}(0, r)
\end{array}\right.
$$

So, the Z-number solution of Eq. (82) is displayed as $[(\mathbf{S}[\underline{\phi}(t, r)], \mathbf{S}[\bar{\phi}(t, r)]), p(0.8,0.9,1)]$ where

$$
\left\{\begin{array}{l}
\mathbf{S}[\phi(t, r)]=\phi(0, r)\left(\frac{1}{B+1}\right) \\
\mathbf{S}[\overline{\bar{\phi}}(t, r)]=\overline{\bar{\phi}}(t, r)\left(\frac{1}{B+1}\right)
\end{array}\right.
$$

By utilizing the inverse Sumudu transform for Z-numbers, we have

$$
\left\{\begin{array}{l}
\phi(t, r)=\phi(0, r) \mathbf{S}^{-1}\left(\frac{1}{B+1}\right) \\
\overline{\bar{\phi}}(t, r)=\overline{\bar{\phi}}(0, r) \mathbf{S}^{-1}\left(\frac{1}{B+1}\right)
\end{array}\right.
$$

where

$$
\left\{\begin{array}{l}
\phi(t, r)=e^{-t} \phi(0, r) \\
\overline{\bar{\phi}}(t, r)=e^{-t} \overline{\bar{\phi}}(0, r)
\end{array}\right.
$$

If the initial condition is taken to be a symmetric triangular Znumber as $\phi(0)=[(-a(1-r), a(1-r)), p(0.8,0.9,1)]$, so

Case 1:

$$
\left\{\begin{array}{l}
\phi(t, r)=e^{t}(-a(1-r)) \\
\bar{\phi}(t, r)=e^{t}(a(1-r))
\end{array}\right.
$$

Case 2:

Table 1. Approximation errors based on Z-numbers

\begin{tabular}{|c|l|l|}
\hline$\alpha$ & \multicolumn{1}{|c|}{ FST } & \multicolumn{1}{|c|}{ Average Euler } \\
\hline 0 & {$[(0.0078,0.0195), \mathrm{p}(0.8,0.86,0.94)]$} & {$[(0.0138,0.0215), \mathrm{p}(0.7,0.8,0.87)]$} \\
\hline 0.2 & {$[(0.0085,0.0169), \mathrm{p}(0.75,0.8,0.9)]$} & {$[(0.0188,0.0286), \mathrm{p}(0.7,0.8,0.87)]$} \\
\hline 0.6 & {$[(0.0058,0.0115), \mathrm{p}(0.8,0.9,1)]$} & {$[(0.0182,0.0198), \mathrm{p}(0.7,0.8,0.92)]$} \\
\hline 0.8 & {$[(0.0091,0.0123), \mathrm{p}(0.7,0.75,0.8)]$} & {$[(0.0148,0.0189), \mathrm{p}(0.6,0.7,0.8)]$} \\
\hline 1 & {$[(0.0132,0.0132), \mathrm{p}(0.7,0.8,0.9)]$} & {$[(0.0710,0.0710), \mathrm{p}(0.6,0.75,0.87)]$} \\
\hline
\end{tabular}

Approximation errors based on Z-numbers are shown in Table 1. These errors are the differences between the exact and the approximation solutions, for two different methods: FST and Average Euler method [38].

The following formula is utilized to transfer the Z-numbers to fuzzy numbers,

$$
\sigma=\frac{\int \phi \pi_{P}(\phi) d \phi}{\int \pi_{P}(\phi) d \phi}
$$

By taking in to consideration $Z=(B, \widetilde{p})=[(0.0078,0.0195)$, $p(0.8,0.86,0.94)]$, we obtain $Z^{\sigma}=[0.0078,0.0195 ; 0.86]$ accordingly $Z^{\prime}=[\sqrt{0.86} 0.0078, \sqrt{0.86} 0.0195]$. Approximation errors based on fuzzy numbers are shown in Table 2.

Figure 2 shows the corresponding error plots based on fuzzy numbers. FST is more accurate than the Average Euler method. Figure 3 and Figure 4 demonstrate the corresponding solution plots based on fuzzy numbers. 
By implementing Z-numbers the degree of reliability of the information can be increased $[39,40]$. The comparison between the Z-number $Z=[(0.0078,0.0195), p(0.8,0.86,0.94)]$ and fuzzy number $[0.0072,0.0180]$ is displayed in Figure 5. It can be seen that the Z-number incorporates with several information, also the solution related to the Z-number is more precise. The membership function regarding the restriction in the $Z$-number is considered to be $\mu_{B_{7}}=[0.0078,0.0195]$. It can be in probability form.

Table 2. Approximation errors based on fuzzy numbers

\begin{tabular}{|c|c|c|}
\hline$\alpha$ & FST & Average Euler \\
\hline 0 & {$[0.0072,0.0180]$} & {$[0.0123,0.0192]$} \\
\hline 0.2 & {$[0.0076,0.0151]$} & {$[0.0168,0.0255]$} \\
\hline 0.6 & {$[0.0055,0.0109]$} & {$[0.0162,0.0177]$} \\
\hline 0.8 & {$[0.0078,0.0106]$} & {$[0.0123,0.0158]$} \\
\hline 1 & {$[0.0118,0.0118]$} & {$[0.0614,0.0614]$} \\
\hline
\end{tabular}

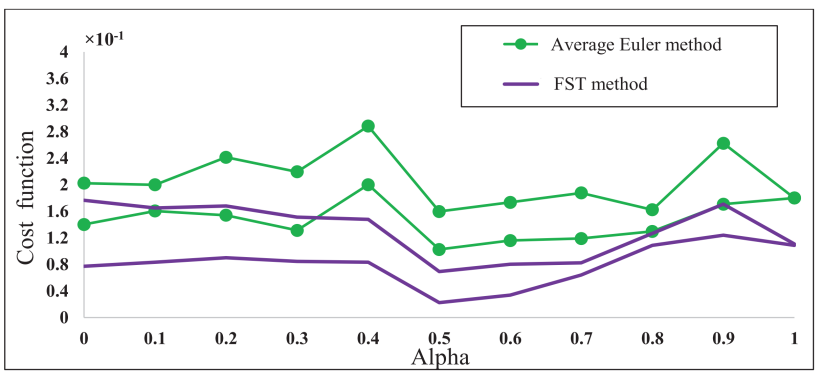

Fig. 2. The lower and upper bounds of absolute errors based on fuzzy numbers

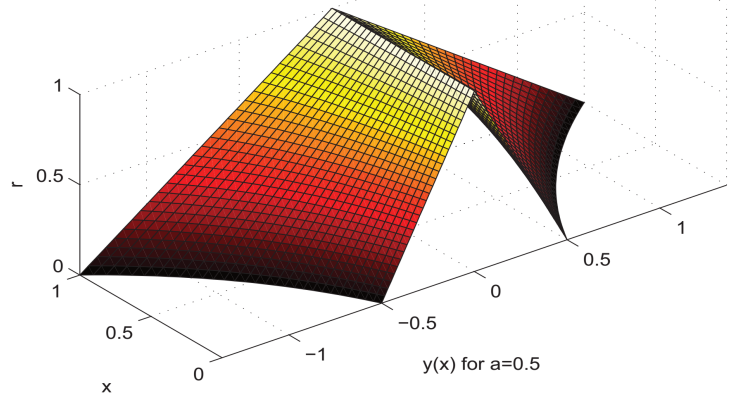

Fig. 3. The solution of FDE under case 1 consideration based on fuzzy numbers

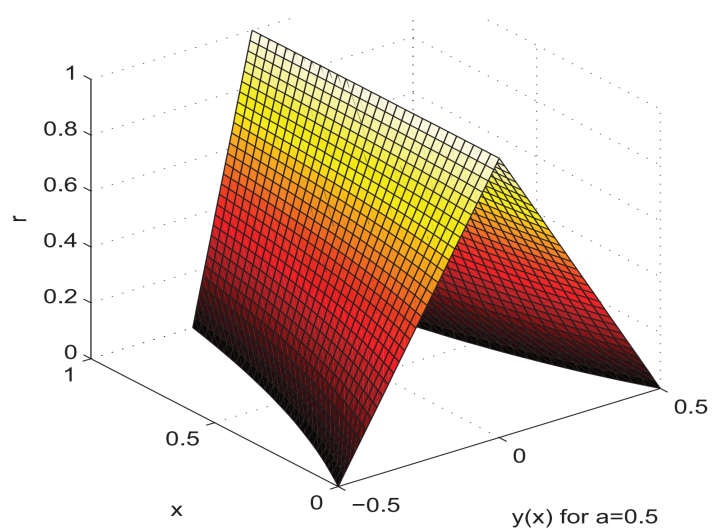

Fig. 4. The solution of FDE under case 2 consideration based on fuzzy numbers

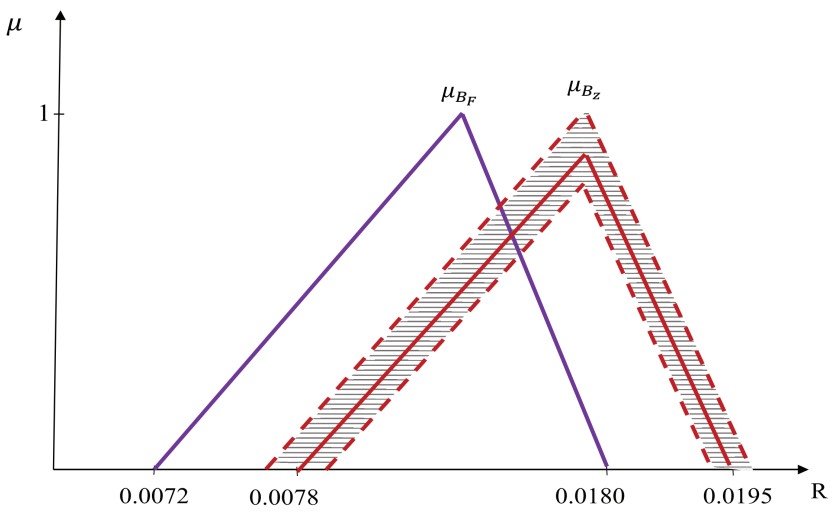

Fig. 5. The comparison between the Z-number and fuzzy number

\subsection{Example 2}

A tank system is displayed in Figure 6. Suppose $I=t+1$ is inflow disturbances of the tank that generates vibration in liquid level $\phi$, also $H=1$ is the flow obstruction, which can be curbed utilizing the valve. $Q=1$ is the cross-section of the tank. The liquid level is illustrated as following relation [41, 42],

$$
\left\{\begin{array}{l}
\phi^{\prime}(t)=-\frac{1}{Q H} \phi(t)+\frac{I}{Q}, \quad 0 \leq t \leq T \\
\phi(0)=[(\underline{\phi}(0, r), \bar{\phi}(0, r)), p(0.85,0.91,1)]
\end{array}\right.
$$

By utilizing the FST method based on Z-number we obtain

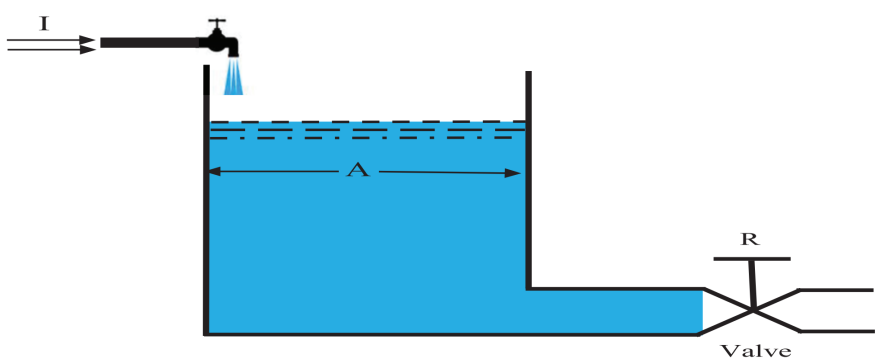

Fig. 6. Liquid tank system

$$
\begin{gathered}
-\mathbf{S}[\varphi(t)]=\left(\frac{1}{B} ? \mathbf{S}[\varphi(t)]\right) ?\left(\frac{1}{B} \mathbf{S}[\varphi(0)]\right) \\
\mathbf{S}\left[\phi^{\prime}(t)\right]\left(\int_{a_{1}}^{\infty} \phi(B t) e^{\prime-t} d t\right.
\end{gathered}
$$

By considering case 2 for Z-numbers the following relation is obtained

$$
\mathbf{S}\left[\varphi^{\prime}(t)\right]=\left(\frac{-1}{B} ? \mathbf{S}[\varphi(t)]\right) ?\left(\frac{-1}{B} \mathbf{S}[\varphi(0)]\right)
$$

So

$$
-\mathbf{S}[\varphi(t)]+\mathbf{S}[t]+\mathbf{S}[1]=\left(\frac{-1}{B} ? \mathbf{S}[\varphi(t)]\right) ?\left(\frac{-1}{B} \mathbf{S}[\varphi(0)]\right)
$$

Based on the Eq. (59), we have 


$$
\left\{\begin{array}{l}
-\mathbf{S}[\underline{\phi}(t, r)]+\mathbf{S}[t]+\mathbf{S}[1]=\frac{1}{B} \mathbf{S}[\underline{\bar{\phi}}(t, r)]-\frac{1}{B} \phi(0, r) \\
-\mathbf{S}[\phi(t, r)]+\mathbf{S}[t]+\mathbf{S}[1]=\frac{1}{B} \mathbf{S}[\phi(t, r)]-\frac{1}{B} \bar{\phi}(0, r)
\end{array}\right.
$$

Therefore, the Z-number solution of Eq. (93) is extracted as

$$
\left\{\begin{array}{c}
\mathbf{S}[\phi(t, r)]=\mathbf{S}[t]+\mathbf{S}[1]+\frac{-1}{B} \mathbf{S}[\phi(t, r)]-\frac{1}{B} \phi(0, r) \\
\mathbf{S}[\bar{\phi}(t, r)]=\mathbf{S}[t]+\mathbf{S}[1]+\frac{1}{B} \mathbf{S}[\overline{\bar{\phi}}(t, r)]-\frac{1}{B} \bar{\phi}(0, r)
\end{array}\right.
$$

hence,

$$
\left\{\begin{array}{l}
\mathbf{S}[\phi(t, r)]=\left(\frac{1}{B+1}\right) \phi(0, r)+B \\
\mathbf{S}[\overline{\bar{\phi}}(t, r)]=\left(\frac{1}{B+1}\right) \overline{\bar{\phi}}(t, r)+B
\end{array}\right.
$$

By utilizing the inverse Sumudu transform for Z-numbers, we obtain

$$
\left\{\begin{array}{l}
\phi(t, r)=\phi(0, r) \mathbf{S}^{-1}\left(\frac{1}{B+1}\right)+\mathbf{S}^{-1}(B) \\
\overline{\bar{\phi}}(t, r)=\overline{\bar{\phi}}(0, r) \mathbf{S}^{-1}\left(\frac{1}{B+1}\right)+\mathbf{S}^{-1}(B)
\end{array}\right.
$$

Where $\left\{\begin{array}{l}\phi(t, r)=e^{-t} \phi(0, r)+t \\ \overline{\bar{\phi}}(t, r)=e^{-t} \overline{\bar{\phi}}(0, r)+t\end{array}\right.$

If the initial condition is taken to be a symmetric triangular $\mathrm{Z}$ number as $\phi(0)=[(-a(1-r), a(1-r)), p(0.85,0.9,1)]$, , so

$$
\left\{\begin{array}{c}
\phi(t, r)=e^{-t}(-a(1-r))+t \\
\bar{\phi}(t, r)=e^{-t}(a(1-r))+t
\end{array}\right.
$$

Approximation errors based on Z-numbers are shown in Table 3. These errors are the differences between the exact and the approximation solutions, for two different methods: FST and Max-Min Euler method [38]. Figure 7 shows the Corresponding solution plot based on fuzzy numbers.

Table 3. Approximation errors based on Z-numbers

\begin{tabular}{|c|l|l|}
\hline$\alpha$ & \multicolumn{1}{|c|}{ FST } & \multicolumn{1}{|c|}{ Max-Min Euler } \\
\hline 0 & {$[(0.0053,0.0128), \mathrm{p}(0.8,0.85,0.9)]$} & {$[(0.0108,0.0195), \mathrm{p}(0.8,0.9,1)]$} \\
\hline 0.2 & {$[(0.0019,0.0127), \mathrm{p}(0.7,0.81,0.9)]$} & {$[(0.0072,0.0138), \mathrm{p}(0.6,0.7,0.8)]$} \\
\hline 0.4 & {$[(0.0031,0.0089), \mathrm{p}(0.8,0.9,1)]$} & {$[(0.0113,0.0123), \mathrm{p}(0.7,0.8,0.91)]$} \\
\hline 0.6 & {$[(0.0008,0.0027), \mathrm{p}(0.8,0.9,1)]$} & {$[(0.0043,0.0078), \mathrm{p}(0.7,0.8,0.9)]$} \\
\hline 0.8 & {$[(0.0049,0.0079), \mathrm{p}(0.7,0.8,0.86)]$} & {$[(0.0069,0.0099), \mathrm{p}(0.6,0.7,0.8)]$} \\
\hline 1 & {$[(0.0109,0.0109), \mathrm{p}(0.7,0.8,0.9)]$} & {$[(0.0587,0.0587), \mathrm{p}(0.6,0.75,0.8)]$} \\
\hline
\end{tabular}

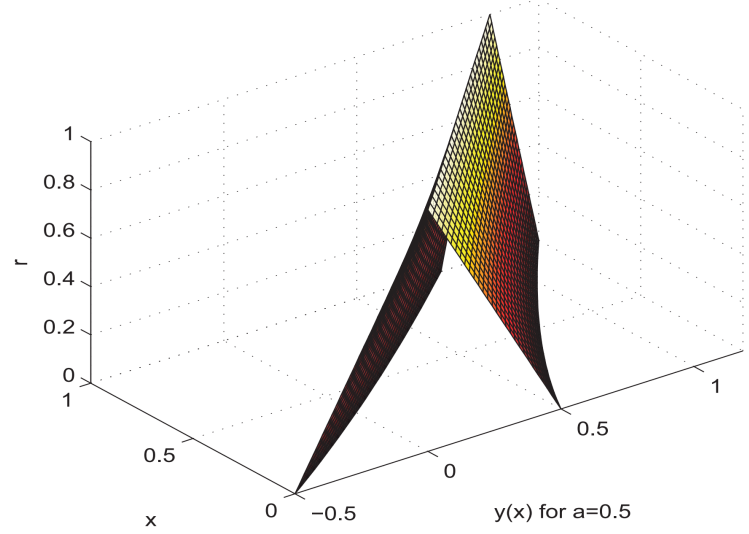

Fig. 7. The solution of FDE under case 2 consideration based on fuzzy numbers

\section{Conclusion}

In this paper, a novel method based on the FST is proposed in order to find the solution of the first order FDEs on the basis of the $\mathrm{Z}$-numbers. The new method is clarified by utilizing the concept of strongly generalized differentiability. By using the FST method, the FDE converts to an algebraic problem. Some essential theorems are laid down in order to demonstrate the properties of the FST. Two real examples are applied to demonstrate the effectiveness of the proposed technique. This work has a significant contribution in initializing a superior starting point for such extensions.

\section{References}

[1] R. Jafari, S. Razvarz, "Solution of Fuzzy Differential Equations using Fuzzy Sumudu Transforms" IEEE International Conference on Innovations in Intelligent Systems and Applications, 84-89, 2017. DOI: 10.1109/INISTA.2017.8001137

[2] D. Zwillinger, "Handbook of differential equations" Gulf Professional Publishing. 1998.

[3] R. Jafari, W. Yu, "Fuzzy Control for Uncertainty Nonlinear Systems with Dual Fuzzy Equations" Journal of Intelligent and Fuzzy Systems. 29(3), pp.1229-1240, 2015. DOI: 10.3233/IFS-151731

[4] R. Jafari, W. Yu, "Fuzzy Differential Equation for Nonlinear System Modeling with Bernstein Neural Networks" IEEE Access., 2017. doi:10.1109/ACCESS.2017.2647920

[5] R. Jafari, W. Yu, "Uncertainty Nonlinear Systems Modeling with Fuzzy Equations" Mathematical problems in Engineering. 2017, 2017. doi: $10.1155 / 2017 / 8594738$

[6] S.L. Chang, L.A. Zadeh, "On fuzzy mapping and control” IEEE Trans. Syst. Man Cybern. 2(1), 30-34, 1972. DOI 10.1109/TSMC.1972.5408553

[7] D. Dubois, H. Prade, "Towards fuzzy differential calculus: part 3 differentiation" Fuzzy Sets Syst. 8(3), 225-233, 1982 https://doi.org/10.1016/S0165-0114(82)80001-8

[8] P. Kloeden, "Remarks on Peano-like theorems for fuzzy differential equations" Fuzzy Set Syst. 44(1), 161-164, 1991. https://doi.org/10.1016/0165-0114(91)90041-N

[9] B. Bede, I.J. Rudas, A.L. Bencsik "First order linear fuzzy differential equations under generalized differentiability" Inform. Sci. 177(7), 16481662, 2007. https://doi.org/10.1016/j.ins.2006.08.021

[10] T. Allahviranloo, M.B. Ahmadi, "Fuzzy Laplace Transform" Soft Computing. 14, 235-243, 2010.

[11] Z. Ding, M. Ma, A. Kandel, "Existence of the solutions of fuzzy differential equations with parameters" Inform Sci. 99(3-4), 205-217, 1997. https://doi.org/10.1016/S0020-0255(96)00279-4

[12] V.A. Truong, V.H. Ngo, D.P. Nguyen, "Global existence of solutions for interval-valued integro-differential equations under generalized $\mathrm{H}$ differentiability" Advances in Difference Equations. 1, 217-233, 2013. https://doi.org/10.1186/1687-1847-2013-217

[13] T. Allahviranloo, N.A. Kiani, M. Barkhor- dari, "Toward the exiistence and uniqueness of solution of second-order fuzzy differential equations" $\begin{array}{lllr}\text { Inform. } & \text { Sci. } & \mathbf{1 7 9}, & 1207-1215,\end{array}$ https://doi.org/10.1016/j.ins.2008.11.004

[14] F. Hawrra, K.H. Amal, "On fuzzy Laplace transform for fuzzy differential equations of the third order" Journal of Kerbala University, 11(3), 251-256, 2013.

[15] L. Stefanini, B. Bede, "Generalized Hukuhara differentiability of interval-valued functions and interval differential equations" $\mathrm{J}$ Nonlinear Anal. (TMA). 71(3-4), 1311-1328, 2009. https://doi.org/10.1016/j.na.2008.12.005

[16] F.B.M. Belgacem, A.A. Karaballi, S.L. Kalla, "Analytical investigations of the Sumudu transform and applications to integral 


\section{S. Razvarz et al. / Advances in Science, Technology and Engineering Systems Journal Vol. 3, No. 1, 66-75 (2018)}

production equations" Math. Prob. Eng. 103(3), 103-118, 2003. http://dx.doi.org/10.1155/S1024123X03207018.

[17] Y. Liu, W. Chen, "A new iterational method for ordinary equations using Sumudu transform" Advances in Analysis, 1(2), 89-94, 2016. DOI: $10.22606 /$ aan.2016.12004

[18] H.M. Srivastava, A. Khalili Golmankhaneh, D. Baleanu, X.J Yang, "Local Fractional Sumudu Transform with Application to IVPs on Cantor Sets" Abstract and Applied Analysis. Article ID 620529, 2014.17, 2014. http://dx.doi.org/10.1155/2014/620529, 2014

[19] F.B.M. Belgacem, A.A. Karaballi, "Sumudu transform fundamental properties investigations and applications" J. Appl. Math. Stoch. Anal. 2006, 1-23, 2006. http://dx.doi.org/10.1155/JAMSA/2006/91083

[20] A. Kiliçman, H. Eltayeb, R.P. Agarwal, "On Sumudu Transform and System of Differential Equations" abstr. Appl. Anal, 2010, 1-11, 2010. doi:10.1155/2010/598702.

[21] N.A. Abdul Rahman, M.Z. Ahmad, "Fuzzy Sumudu transform for solving fuzzy partial differential equations" J. Nonlinear Sci. Appl. 9, 3226-3239, 2016.

[22] Q.D. Katatbeh, F.B.M. Belgacem, "Applications of the Sumudu transform to fractional differential equations" Nonlinear Studies. 18(1), 99-112, 2011.

[23] L.A. Zadeh, "Generalized theory of uncertainty (GTU)-principal concepts and ideas" Computational Statistics and Data Analysis, 51(1), 15-46, 2006. https://doi.org/10.1016/j.csda.2006.04.029

[24] B. Kang, D. Wei, Y. Li, Y. Deng, "A method of converting Z-number to classical fuzzy number" Journal of Information and Computational Science, 9(3), 703-709, 2012.

[25] L.A. Gardashova, "Application of operational approaches to solving decision making problem using Z-Numbers" Journal of Applied Mathematics, 5(9), 1323-1334, 2014. DOI: 10.4236/am.2014.59125

[26] R.A. Aliev, A.V. Alizadeh, O.H. Huseynov, "The arithmetic of discrete Z-numbers" Inform. Sci. 290, 134-155, 2015. https://doi.org/10.1016/j.ins.2014.08.024

[27] B. Kang, D. Wei, Y. Li, Y. Deng, "Decision making using Z-Numbers under uncertain environment" Journal of Computational Information Systems, 8(7), pp.2807-2814, 2012.

[28] A. Jafarian, R. Jafari, M. Mohamed Al Qurashi, D. Baleanud, “A novel computational approach to approximate fuzzy interpolation polynomials" Springer Plus, 5, pp.14-28, 2016. https://doi.org/10.1186/s40064-016-3077-5

[29] R. Jafari, W. Yu, X. Li, "Numerical Solution of Fuzzy Equations with Z-numbers using Neural Networks" Intelligent Automation and Soft Computing, 2017. doi: 10.1080/10798587.2017.1327154.

[30] M.L. Puri, D. Ralescu, "Fuzzy random variables" J. Math. Analysis Appl. 114(2), 409-422, $1986 . \quad$ https://doi.org/10.1016/0022247X(86)90093-4

[31] H.-C. Wu, "The improper fuzzy Riemann integral and its numerical integration" Inform. Sci. 111(1-4), 109-137, 1999. https://doi.org/10.1016/S0020-0255(98)00016-4

[32] B. Bede, S.G. Gal, "Generalizations of the differentiability of fuzzynumber-valued functions with applications to fuzzy differential equations" Fuzzy Set Syst. 151(3), 581-599, 2005. https://doi.org/10.1016/j.fss.2004.08.001

[33] Y. Chalco-Cano, H. Roman-Flores, "On new solutions of fuzzy differential equations" Chaos Solitons Fractals. 38(1), 112-119, 2006. https://doi.org/10.1016/j.chaos.2006.10.043

[34] L.A. Zadeh, "Toward a generalized theory of uncertainty (GTU) an outline" Inform. Sci. 172 (1-2), 1-40, 2005. https://doi.org/10.1016/j.ins.2005.01.017

[35] R.A. Aliev, W. Pedryczb, V. Kreinovich, O.H. Huseynov, "The general theory of decisions" Inform. Sci. 327(10), 125-148, 2016. https://doi.org/10.1016/j.ins.2015.07.055

[36] R.A. Aliev, O.H. Huseynov, R.R. Aliyev, A.V. Alizadeh, "The arithmetic of Z-numbers" Theory and applications World Scientific, Singapore, 2015.
[37] R.H. Pletcher, J.C. Tannehill, D. Anderson, "Computational Fluid Mechanics and Heat Transfer", Taylor and Francis, 1997.

[38] S. Tapaswini, S. Chakraverty, "Euler-based new solution method for fuzzy initial value problems" Int. J. Artificial. Intell. Soft. Comput. 4(1), 58-79, 2014. https://doi.org/10.1504/JJAISC.2014.059288

[39] S. Razvarz, M. Tahmasbi, "Fuzzy equations and Z-numbers for nonlinear systems control”, Procedia Computer Science, 120, 923-930, 2017. https://doi.org/10.1016/j.procs.2017.11.327

[40] R. Jafari, W. Yu, "Uncertain nonlinear system control with fuzzy differential equations and Z-numbers", 18th IEEE International Conference on Industrial Technology, Canada, 890-895, doi:10.1109/ICIT.2017.7915477, 2017.

[41] V.L. Streeter, E.B. Wylie, K.W. Bedford, "Fluid mechanics" New York: McGraw Hill, 1998.

[42] R. Jafari, W. Yu, X. Li, S. Razvarz, "Numerical Solution of Fuzzy Diderential Equations with Z-numbers Using Bernstein Neural Networks" International Journal of Computational Intelligence Systems, 10, 1226-1237, 2017. doi:10.2991/ijcis.10.1.81 